Claremont Colleges

Scholarship@ Claremont

All HMC Faculty Publications and Research

HMC Faculty Scholarship

9-1-1999

\title{
Stability of Self-similar Solutions for Van der Waals Driven Thin Film Rupture
}

Thomas P. Witelski

Duke University

Andrew J. Bernoff

Harvey Mudd College

\section{Recommended Citation}

Stability of self-similar solutions for van der Waals driven thin film rupture. Thomas P. Witelski and Andrew J. Bernoff, Phys. Fluids 11, 2443 (1999).

This Article is brought to you for free and open access by the HMC Faculty Scholarship at Scholarship @ Claremont. It has been accepted for inclusion in All HMC Faculty Publications and Research by an authorized administrator of Scholarship @ Claremont. For more information, please contact scholarship@cuc.claremont.edu. 


\title{
LETTERS
}

The purpose of this Letters section is to provide rapid dissemination of important new results in the fields regularly covered by Physics of Fluids. Results of extended research should not be presented as a series of letters in place of comprehensive articles. Letters cannot exceed three printed pages in length, including space allowed for title, figures, tables, references and an abstract limited to about 100 words. There is a three-month time limit, from date of receipt to acceptance, for processing Letter manuscripts. Authors must also submit a brief statement justifying rapid publication in the Letters section.

\section{Stability of self-similar solutions for van der Waals driven thin film rupture}

\author{
Thomas P. Witelski \\ Department of Mathematics, Duke University, Durham, North Carolina 27708 \\ Andrew J. Bernoff \\ Department of Mathematics, Harvey Mudd College, Claremont, California 91711
}

(Received 1 March 1999; accepted 14 May 1999)

\begin{abstract}
Recent studies of pinch-off of filaments and rupture in thin films have found infinite sets of first-type similarity solutions. Of these, the dynamically stable similarity solutions produce observable rupture behavior as localized, finite-time singularities in the models of the flow. In this letter we describe a systematic technique for calculating such solutions and determining their linear stability. For the problem of axisymmetric van der Waals driven rupture (recently studied by Zhang and Lister), we identify the unique stable similarity solution for point rupture of a thin film and an alternative mode of singularity formation corresponding to annular "ring rupture." (C) 1999 American Institute of Physics. [S1070-6631(99)03609-0]
\end{abstract}

Several recent studies have found infinite sets of similarity solutions to problems involving pinch-off and rupture. ${ }^{1-4}$ Here we study a model described in a recent paper of Zhang and Lister, ${ }^{2}$ who examine the rupture of a thin liquid film on a substrate due to van der Waals forces. The evolution of the thickness of the viscous film, $h=h(\mathbf{x}, t)$, under the influence of van der Waals forces and surface tension is given by the lubrication equation,

$$
\frac{\partial h}{\partial t}=-\nabla \cdot\left(h^{-1} \nabla h\right)-\nabla \cdot\left(h^{3} \nabla \nabla^{2} h\right) .
$$

Similar fourth-order nonlinear diffusion equations describing thin films with surface tension have been studied extensively. ${ }^{5-8}$ Zhang and Lister address the problem of rupture in this equation, associated with a singularity forming with $h \rightarrow 0$ at a finite time, $t_{c}$. As rupture is approached, some of the assumptions used to derive (1) from the NavierStokes equations may break down, but within this model, formation of the singularity is self-consistent. From dimensional analysis, Zhang and Lister show that first-type similarity solutions exist for rupture with $h=O\left(\left(t_{c}-t\right)^{1 / 5}\right)$ and $x=O\left(\left(t_{c}-t\right)^{2 / 5}\right)$ as $t \rightarrow t_{c}$ for both planar line rupture and axisymmetric point rupture. Using these scalings, they reduce (1) to a fourth-order nonlinear ordinary differential equation (ODE) for the self-similar profiles. The beginnings of a discrete, countably infinite set of these solutions was found using a numerical shooting method. ${ }^{1,3}$ The first of these profiles was also observed in direct simulations of the partial differential equation (PDE) (1). Here, we present an alternative numerical approach for calculating the selfsimilar profiles, as solutions of a nonlinear two-point boundary value problem, using Newton's method. This approach has the advantage of yielding the linear stability of these solutions. We show that only the fundamental point rupture solution is linearly stable to axisymmetric perturbations; we also show that it is stable to non-axisymmetric perturbations.

As in Zhang and Lister, ${ }^{2}$ we consider both the problems of planar, $h=h(x, t)$, and axisymmetric, $h=h(r, t)$, rupture. Motivated by dimensional analysis, we write the solution in terms of similarity variables as

$$
h(r, t)=\tau^{1 / 5} H(\eta, s),
$$

where $\tau=t_{c}-t$ is the time until rupture, and the new spatial and temporal similarity variables are

$$
\eta=r / \tau^{2 / 5}, \quad s=-\ln \tau .
$$

Then (1) is written as a similarity PDE,

$$
\frac{\partial H}{\partial s}=\mathcal{T}(H)+\mathcal{V}(H)+\mathcal{S}(H),
$$

where

$$
\mathcal{T}(H) \equiv \frac{1}{5}\left(H-2 \eta H_{\eta}\right)
$$

is a linear operator resulting from the transformation of the time derivative, $h_{t}$, and

$$
\mathcal{V}(H) \equiv-\eta^{1-d}\left(\eta^{d-1} H^{-1} H_{\eta}\right)_{\eta}
$$

is the axisymmetric $(d=2)$ or planar $(d=1)$ form of the van der Waals term, $-\nabla \cdot\left(h^{-1} \nabla h\right)$, and

$$
\mathcal{S}(H) \equiv-\eta^{1-d}\left(\eta^{d-1} H^{3}\left[\eta^{1-d}\left\{\eta^{d-1} H_{\eta}\right\}_{\eta}\right]_{\eta}\right)_{\eta}
$$

corresponds to the surface tension term, $-\nabla \cdot\left(h^{3} \nabla \nabla^{2} h\right)$. 
In this notation, self-similar profiles are steady, $s$-independent solutions, $H=\bar{H}(\eta)$, of the fourth-order nonlinear ODE

$$
\mathcal{N}_{4}(\bar{H}) \equiv \mathcal{T}(\bar{H})+\mathcal{V}(\bar{H})+\mathcal{S}(\bar{H})=0 .
$$

To describe localized self-similar behavior, solutions of (8) must also satisfy far-field boundary conditions allowing asymptotic matching to a slowly varying outer solution. The structure of the solution away from a localized singularity is independent of the evolution of the singularity as the critical time is approached, $t \rightarrow t_{c} \cdot{ }^{2,4}$ For the rupture problem this means that $h_{t}$ must remain bounded (independent of $\tau$ ) at any fixed distance in $r$ from the rupture point. In similarity variables, this restriction transforms into a far-field Robin boundary condition for the self-similar solutions,

$$
\mathcal{T}(\bar{H})=\frac{1}{5}\left(\bar{H}-2 \eta \bar{H}_{\eta}\right) \rightarrow 0 \quad \text { as } \quad|\eta| \rightarrow \infty .
$$

These far-field asymptotics force the similarity solutions to have the structure

$$
\bar{H} \sim A \eta^{1 / 2} \quad \text { as } \quad|\eta| \rightarrow \infty,
$$

where the positive matching constant $A$ provides a measurable global parameter for comparison of different solutions of (1). As described by Zhang and Lister, ${ }^{2}$ specifying this asymptotic behavior as the far-field boundary yields locally unique solutions to (8).

Due to the high order and nonlinearity of (8), analytic results are severely limited and numerical methods must be used to obtain solutions. Like other studies of similarity solutions for thin film problems, ${ }^{1,3}$ Zhang and Lister used a two-parameter shooting method to search for solutions satisfying (10) starting from given values for $\bar{H}(0), \bar{H}_{\eta \eta}(0)$ at $\eta=0$. In contrast, we obtained uniformly accurate solutions of (8) from a finite difference scheme, on a finite but large domain of length $L$, with boundary conditions (9), solved via an under-relaxed Newton's method. The boundary conditions are imposed at each edge, ${ }^{4}$ and the domain is increased until the numerical solution is independent of $L$. Such iterative methods are only locally convergent in solution space and therefore need well-chosen starting estimates.

For our initial estimate of the solution, we construct a longwave approximation, $\widetilde{H}(\eta)$, to (8) and (9) in which the fourth-order term, $\mathcal{S}(H)$, is assumed to be subdominant. The resulting second-order problem is more analytically and numerically tractable. Let $\widetilde{H}(\eta)=\widetilde{H}^{(0)}(\eta)+\widetilde{H}^{(1)}(\eta)+\cdots$, where $\widetilde{H}^{(0)}(\eta)$ is given by

$$
\mathcal{N}_{2}\left(\widetilde{H}^{(0)}\right) \equiv \mathcal{T}\left(\widetilde{H}^{(0)}\right)+\mathcal{V}\left(\widetilde{H}^{(0)}\right)=0 .
$$

Equation (11) has a unique solution for every positive value of $A$ in the asymptotic boundary conditions (10). Subsequently, $\widetilde{H}^{(1)}(\eta)$ is given by the solution of the linear second-order problem

$$
\mathcal{L}_{2}\left(\widetilde{H}^{(0)}\right) \widetilde{H}^{(1)}=-\mathcal{S}\left(\widetilde{H}^{(0)}\right),
$$

where $\mathcal{L}_{2} \equiv \delta \mathcal{N}_{2}$ is the linearized second-order operator. The longwave approximations, $\widetilde{H}(\eta)$, generated by this approach are parametrized by the far-field constant $A$ in (10).
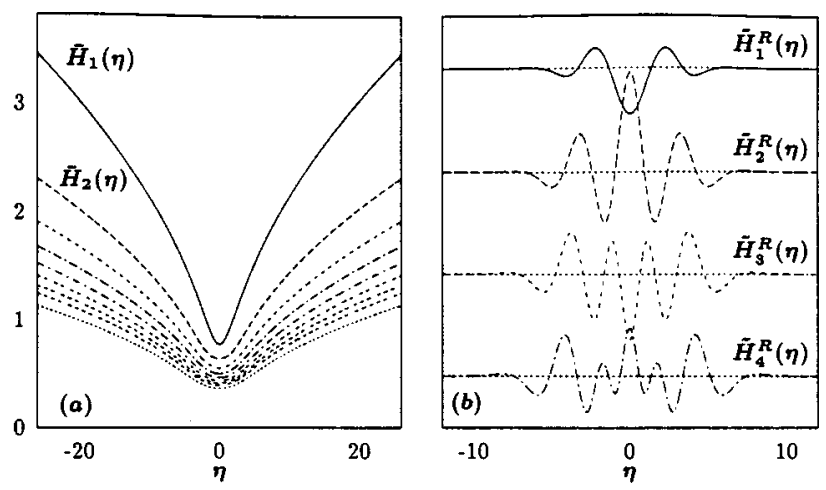

FIG. 1. (a) The first ten solution profiles for finite-time self-similar axisymmetric point rupture, $\bar{H}_{m}(\eta), m=1,2, \ldots, 10$. (b) The longwave residuals, $\bar{H}_{m}^{R}(\eta)$, scaled by $O\left(A_{m}^{8}\right)$, showing the fine-scale differences between the $\bar{H}_{m}(\eta)$ solutions for the first four profiles.

For almost every value of $A>0$, the iterative scheme for solving (8) and (9) starting with the two-term approximation to $\widetilde{H}(\eta)$ converges to one of the discrete self-similar solutions $\bar{H}_{m}(\eta), m=1,2, \ldots$ [see Fig. 1(a)], the first six of which were found by Zhang and Lister. ${ }^{2}$ Each of these $\bar{H}_{m}$ solutions has a distinct value of $A=A_{m}$, with a finite basin of attraction for the iterative scheme for $A$ in a neighborhood of $A_{m}$. Having found the first 25 solutions for both the planar and axisymmetric cases, we note that the $A_{m}$ constants closely follow power law scalings, $A_{m} \approx 0.60 / \mathrm{m}^{0.43}$ for $d$ $=2$ and $A_{m} \approx 0.75 / m^{0.46}$ for $d=1$. In addition to the values of $A_{m}$, Zhang and Lister distinguished the different solutions they found by tabulating $\bar{H}(0)$ and $\bar{H}_{\eta \eta}(0)$ and plotting $\bar{H}_{\eta \eta \eta}(\eta)$ for each. An alternative criterion for differentiating these solutions is to examine the residual between the exact solution and longwave approximation, $\bar{H}_{m}^{R}(\eta)=\bar{H}_{m}(\eta)$ $-\widetilde{H}_{m}(\eta)$ [see Fig. 1(b)]. The residuals reveal small amplitude oscillatory corrections localized to a neighborhood of $\eta=0$, a contribution to the solution missed by the longwave expansion. They are eigenstates of a fourth-order boundary value problem with the values of $A_{m}$ serving the role of eigenvalues. Observe that each successive $\bar{H}_{m}^{R}(\eta)$ gains an extra pair of zeroes, yielding a well-defined method for counting of the $\bar{H}_{m}(\eta)$ solutions, independent of, and consistent with, the $A_{m}$ values. Note that in the limit $A \rightarrow 0$, $\mathcal{S}(\widetilde{H})$ is indeed subdominant, justifying our longwave expansion. Using the first two terms in this expansion was sufficiently accurate to yield convergence of Newton's method.

We will now use linear stability analysis to show that only the fundamental self-similar solution $\bar{H}_{1}(\eta)$, for $d$ $=1,2$, is stable, verifying the observations of the direct PDE simulations. ${ }^{2}$ Consider $s$ time-dependent similarity solutions of (4) given by infinitesimal perturbations of the self-similar solutions,

$$
H(\eta, s)=\bar{H}_{m}(\eta)+\hat{H}(\eta) e^{\lambda s} .
$$

Substituting (13) into (4) yields the linearized eigenvalue problem

$$
\lambda \hat{H}=\mathcal{L}_{4}\left(\bar{H}_{m}\right) \hat{H},
$$




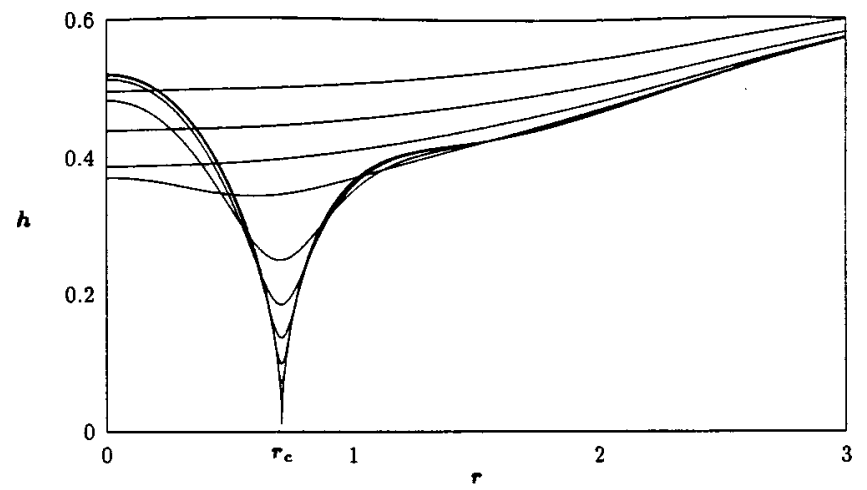

FIG. 2. Solution of the axisymmetric problem for (1) showing destabilization of the $\bar{H}_{4}(\eta)$ solution yielding ring rupture with $r_{c} \approx 0.71$.

where the fourth-order linearized operator is given by $\mathcal{L}_{4}$ $=\delta \mathcal{N}_{4}$. The far-field boundary conditions, $\mathcal{T}(\hat{H})=\lambda \hat{H}$ as $|\eta| \rightarrow \infty$, again follow from the spatial localization of the singularity. ${ }^{4}$ The numerical solution of this problem follows directly from our use of Newton's method to obtain the nonlinear solutions $\bar{H}_{m}(\eta)$. The converged Jacobian matrix is the numerical representation of the linearized operator $\mathcal{L}_{4}\left(\bar{H}_{m}\right)$; its eigenmodes are calculated via inverse iteration. For the axisymmetric problem $(d=2)$ we also calculated stability of the $\bar{H}_{m}(\eta)$ solutions with respect to nonaxisymmetric perturbations, $\hat{H}(\eta) e^{i n \theta} e^{\lambda s}$, by retaining the original $\theta$ dependence of the gradients in (1).

The symmetries of PDE (1) must be examined to correctly interpret the spectrum of the linear stability problem. ${ }^{4}$ A continuous one-parameter family of rupture solutions of (1) exists under time translations of the rupture time, $t_{c}$ $\rightarrow t_{c}+\epsilon$. Linearizing the action of this symmetry yields an eigenmode associated with time translation, $\hat{H}_{T}(\eta)=\mathcal{T}(\bar{H})$ with $\lambda_{T}=1$. Similarly in the planar case $(d=1)$, invariance under spatial translation, $x \rightarrow x+\epsilon$, yields an additional eigenmode, $\hat{H}_{X}(\eta)=\bar{H}_{\eta}$ with $\lambda_{X}=\frac{2}{5}$. For axisymmetric point rupture $(d=2)$, this spatial translation mode describes shifting the rupture point in the $(x, y)$ plane and yields an $n=1$ non-axisymmetric eigenmode. We note that while these symmetry modes have positive eigenvalues, they do not represent genuine instabilities. They reflect the fact that solutions with different rupture positions or critical times appear to be exponentially diverging in the similarity variables, $(\eta, s)$.

Our calculations have shown that, apart from the symmetry modes, only the fundamental solution, $\bar{H}_{1}(\eta)$, for both $d=1,2$, has no positive eigenvalues and hence is stable to linear perturbations. Thus, the $\bar{H}_{1}(\eta)$ solutions describe stable finite-time self-similar rupture. The dynamical stability of these solutions was verified by numerical simulations of the full PDE (1) in both planar and axisymmetric geometries. Our calculations also show that axisymmetric rupture given by $\bar{H}_{1}(\eta)$ is also stable with respect to nonaxisymmetric perturbations for $n=1,2, \ldots$.

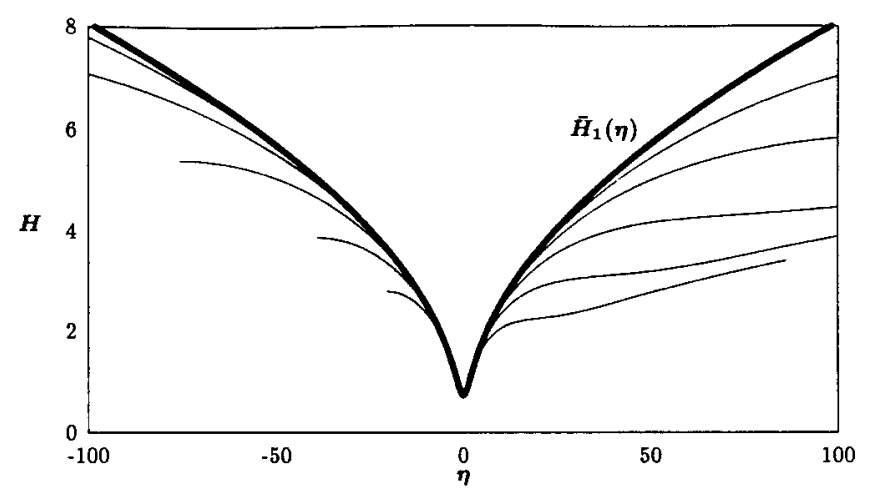

FIG. 3. Rescaled solutions from Fig. 2 showing convergence to the planar $(d=1)$ similarity solution, $\bar{H}_{1}(\eta)$, in terms of the ring-centered similarity variable $\eta=\left(r-r_{c}\right) / \tau^{2 / 5}$.

While the higher-order solutions, $\bar{H}_{m}(\eta)$ are unstable for $m>1$, this does not mean that they do not play a role in the dynamics of rupture. In Fig. 2, we show a direct numerical solution of the axisymmetric PDE (1) starting from initial data given by $\bar{H}_{4}(r)$, which reveals a new scenario for rupture. The similarity solution soon destabilizes and rupture does not take place at the origin, $r=0$, but in an annular ring with a finite radius $r_{c}>0$. In Fig. 3, the solutions of the PDE are rescaled in the neighborhood of the ring rupture radius, $r_{c}$. They converge to the stable planar $(d=1)$ rupture similarity solution, $\bar{H}_{1}(\eta)$; this reflects the fact that the influence of the ring curvature can be neglected in the localized neighborhood of $r_{c}$ as $t \rightarrow t_{c}$. In a full simulation of (1), we expect ring rupture to be unstable to non-axisymmetric perturbations, leading to a set of isolated point ruptures; further investigation of these phenomena awaits forthcoming work.

\section{ACKNOWLEDGMENTS}

We are indebted to Wendy Zhang and John Lister for sharing a preprint ${ }^{2}$ of their work with us, and to Andrea Bertozzi for enlightening conversations about thin films and self-similarity.

${ }^{1}$ M. P. Brenner, J. R. Lister, and H. A. Stone, "Pinching threads, singularities and the number 0.0304 ... ,', Phys. Fluids 8, 2827 (1996).

${ }^{2}$ W. W. Zhang and J. R. Lister, "Similarity solutions for van der Waals rupture of a thin film on a solid substrate," Phys. Fluids 11, 2454 (1999).

${ }^{3}$ D. Vaynblat, M. P. Brenner, J. R. Lister, and T. P. Witelski, "Rupture of thin viscous films by van der Waals Forces," preprint (1999).

${ }^{4}$ A. J. Bernoff, A. L. Bertozzi, and T. P. Witelski, "Axisymmetric surface diffusion: Dynamics and stability of self-similar pinchoff," J. Stat. Phys. 93, 725 (1998).

${ }^{5}$ A. Oron, S. H. Davis, and S. G. Bankoff, "Long-scale evolution of thin liquid films," Rev. Mod. Phys. 69, 931 (1997).

${ }^{6} \mathrm{~J}$. Eggers, "Nonlinear dynamics and breakup of free-surface flows,"' Rev. Mod. Phys. 69, 865 (1997).

${ }^{7}$ T. G. Myers, “Thin films with high surface tension," SIAM Rev. 40, 441 (1998).

${ }^{8}$ A. L. Bertozzi and M. Pugh, "The lubrication approximation for thin viscous films: the moving contact line with a "porous media" cut-off of van der Waals interactions," Nonlinearity 7, 1535 (1994). 